\title{
Supuestos y filiaciones filosóficas de los paradigmas astronómicos en el virreinato del Perú
}

\author{
Roberto Katayama Omura \\ Universidad Nacional Mayor de San Marcos \\ rjkatayamao@hotmail.com
}

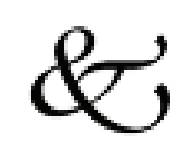

\begin{abstract}
Resumen
En el presente artículo se estudian los supuestos y filiaciones filosóficonaturales de los paradigmas astronómicos vigentes en el virreinato del Perú. Se busca demostrar que hasta el siglo XVIII fue dominante el paradigma clásico o geocéntrico en la versión moderna de Tycho Brahe y que también estuvo presente el paradigma clásico en su versión tradicional o ptolemaica aunque con menos fuerza. Finalmente, se propone que desde mediados del siglo XVIII se introduce en el virreinato peruano el paradigma moderno kepleriano-newtoniano el cual se impondrá a fines del mismo siglo.
\end{abstract}

Palabras claves: Filosofía, virreinato, cosmógrafos, geocéntrico, ptolemaico, ticónico.

\begin{abstract}
In this paper we study the variety of astronomic paradigms in Peru's viceroyalty and its background of natural philosophy. In this study we show that until the xviii century, Tycho Brahe's vertion of the classic geocentric paradigm was the dominant. The Ptolemaic paradigm was present, too. But its important was not very relevant. Since the middle of xviii century the modern paradigm of Kepler and Newton arrives to Peruvian viceroyalty. For the end of xviii century that paradigm was the dominant.
\end{abstract}

Keywords: Philosophy, Viceroyalty, Cosmographer, Geocentric, Ptolemaic, Tyconic. 


\section{Reconfigurando el paradigma historiográfico}

Como es de unánime acuerdo entre los historiadores de la ciencia, la llamada "revolución copernicana" no sólo significó un cambio en nuestra concepción del sistema planetario (paso del geocentrismo al heliocentrismo) sino que a partir de este cambio en el paradigma astronómico se desencadenaron otros de igual o mayor importancia:

"La revolución copernicana fue una revolución en el campo de las ideas, una transformación del concepto del universo que tenía el hombre hasta aquel momento y de su propia relación con el mismo" (Kuhn, 1978:23)

De este modo, comprender las ideas astronómicas vigentes en una comunidad científica determinada es una manera de comprender las concepciones del universo, los compromisos ontológicos y la visión del mundo existente en esa misma época. ¿Cuáles fueron, entonces, los paradigmas astronómicos en el virreinato peruano?

En el caso de los estudios sobre la historia de las ideas astronómicas en el Perú virreinal, el punto de vista historiográfico ha oscilado entre dos extremos, tenemos, por un lado, el de aquellos que sostienen que esta no existió, y basan su argumentación en la supuesta inexistencia del instrumental idóneo (Estremadoyro, 1986:37) por otro lado, tenemos el de aquellos que sostienen que sí la hubo y que se trató de una lucha titánica por parte de las mentes científicas peruanas de la época por introducir las ideas científicas modernas galileanonewtonianas en nuestro medio, a pesar de la oposición del escolasticismo dominante. Al respecto sostuvo Augusto Salazar Bondy:

"La difusión de las nuevas ideas se realizó sin embargo, a lo largo del siglo XVIII y en lucha abierta contra los escolásticos que dominaban las universidades y colegios." (Salazar, 1967:27)

Esta segunda corriente historiográfica llega a describir al científico virreinal peruano de élite como "un espíritu abierto a las formas modernas del saber científico y de sistemas como los de Copérnico, Gassendi y Descartes, de los cuales se sirve para criticar la doctrina escolástica” (Rivara, 2000:255)

¿Qué tan ciertas son estas opiniones? Nuestra participación por más de una década en un grupo de investigación de la Universidad Nacional Mayor de San Marcos acerca del pensamiento filosófico y científico virreinal peruano, nos ha permitido determinar que ninguna de estas lecturas son exactas; es cierto que hubo astronomía en el virreinato peruano, es cierto también que la élite científica peruana estaba al tanto de los últimos avances en filosofía y ciencia europeos, pero no necesariamente eran mentes ilustradas luchando contra el oscurantismo medieval virreinal ni tampoco necesariamente estaban de acuerdo con las ideas dominantes en Europa. 
Mientras la élite científica astronómica europea ya estaba trabajando una "ciencia normal" inmersa en el paradigma cuantitativo, nominalista y mecanicista galileano-newtoniano. La élite científica peruana, pese a conocer los últimos avances y descubrimientos europeos, lo hacía bajo un paradigma diferente al europeo dominante. ¿Cuál era este paradigma?, ¿cuáles eran sus alcances, sus supuestos y sus implicancias?

Las reflexiones astronómicas llevadas a cabo por la élite científica virreinal peruana estaban ligadas o bien a aspectos eminentemente prácticos como las cartas de navegación, la meteorología y el calendario, o bien a fenómenos astronómicos que suscitaban la atención de la población en general y de los científicos en particular como, por ejemplo, lo cometas.

En ese sentido, hemos podido establecer preliminarmente que, a diferencia de los astrónomos europeos de élite, los mejores representantes de la astronomía virreinal peruana no estaban interesados sobre los movimientos planetarios y los fenómenos astronómicos per se sino por la utilidad que ellos pudieran tener para la confección de cartas náuticas, calendarios, estudios del clima (día más cálido, nivel promedio de precipitaciones, etc.), predicción de eclipses, explicación de fenómenos astronómicos como los cometas, etc.

Es en los estudios de estos temas acerca del cual los más grandes astrónomos peruanos de la época escribieron sendas obras. En ellas presentaban sus tesis sobre la naturaleza de los planetas, los satélites y las estrellas así como sus concepciones sobre el cielo y los fenómenos celestes. A partir de este estudio reconstruiremos el sistema del mundo implícito en sus reflexiones para poder determinar los paradigmas astronómicos vigentes en el periodo que nos ocupa.

Cabe señalar que todos estos astrónomos cuyas ideas estudiaremos, con excepción de Joseph Eusebio de Llano y Zapata, desempeñaron también el cargo de Cosmógrafo Mayor del Reino del Perú, por lo que antes de pasar a estudiarlos presentaremos el origen de dicho cargo y las funciones que debían de realizar quienes lo ostentaban.

\section{El Cosmógrafo Mayor del Reino}

\subsection{El Cosmógrafo Mayor del Reino en España}

Una vez que los españoles arribaron a las costas americanas e iniciaron la conquista del continente, la navegación de altura cobró un papel relevante no sólo por temas de exploración y control territorial sino también por temas de navegación ultramarina, seguridad, dominio de los mares e interconexión entre la América española y la península ibérica. Dentro de este contexto las cartas de navegación y el estudio del cielo jugaron un papel preponderante en una época donde las estrellas eran el único punto de orientación en los mares 
y en donde la vida de los pasajeros y el desarrollo del comercio ultramarino dependían de la habilidad de los pilotos de las naos.

Conscientes de la importancia estratégica de tener pilotos competentes, por disposición real se crea en España el cargo de "Piloto Mayor", siendo el primero en ostentar dicho cargo Américo Vespucio desde el 22 de marzo de 1507. Las funciones principales del "Piloto Mayor" eran las de "examinar y graduar a los pilotos, censurar las cartas e instrumentos de navegación, predecir las fases de la Luna, calcular los eclipses y ordenar las tablas de cosmografía" (Ortiz, 1997: 370)

Debido al incremento de las exploraciones y a la importancia tanto de los mapas de los nuevos territorios así como de las rutas marítimas hacia éstos, a partir de 1519 se crea el cargo de "Maestro de Hacer Cartas" y recae en Nuño García de Torres, siendo la principal función de quien ejerciera dicho cargo, el levantar cartas geográficas de los nuevos territorios así como elaborar cartas de navegación.

Con el tiempo los dos cargos son unificados, creándose el cargo de "Cosmógrafo mayor y maestro de hacer Cartas", siendo Diego Ribero el primero en ocupar dicho puesto.

Como las exigencias de la navegación ultramarina se hacían cada vez mayores y se requería por tanto más personal entrenado ex profeso para dicha labor, partir de 1552 se crea en Sevilla la Cátedra de Arte de Navegación y Cosmografía con el fin de preparar primero y examinar después a los futuros pilotos de navíos ultramarinos. (Ortiz, 1997: 371)

\subsection{Los Cosmógrafos Mayores del Reino del Perú}

Debido al incremento del tráfico ultramarino, la demanda de pilotos fue aumentando y llegó a ser tanta (sobre todo en América) que los graduados en España no lograban satisfacerla por lo que muchas veces se recurría en nuestro continente a pilotos sin las adecuadas competencias, lo que ocasionaba naufragios debidos a la impericia. Por ello, durante el gobierno del Príncipe de Esquilache (1615-1621) en el virreinato del Perú se crea el cargo de "Cosmógrafo Mayor del Reino" con el mismo objetivo que el de su contraparte hispánica; preparar y examinar pilotos para la navegación de altura así como el de confeccionar mapas, elaborar cartas de navegación, efectuar observaciones astronómicas, etc.

En el siglo XVII cinco fueron los que ostentaron el cargo de Cosmógrafo Mayor del Reino: Lucas de Quirós (1618-1634), Francisco de Quirós (1619-1645), Diego de León (1645-1661), Francisco Ruiz Lozano (1662-1677) y Juan Ramón Connig (1678-1708). (Ortiz, 1997: 371) 
Es a partir de la época de Ruiz Lozano que el cargo de Cosmógrafo Mayor del Reino se une con el de Catedrático de Prima de Matemáticas debido a que la navegación, la astronomía y el cálculo matemático iban unidos por lo era necesario complementar ambos.

Federico Schwab en su célebre artículo "Los Almanaques Peruanos", nos informa que durante el resto de periodo virreinal (siglo XVIII y las dos primeras décadas del siglo XIX) se sucedieron en el cosmografiato, Pedro de Peralta Barnuevo (1709-1743), José de Mosquera y Villaroel (1744-1749), Juan Rer (17501756), Cosme Bueno (1757-1798), Gabriel Moreno (1799-1809), José Gregorio Paredes (1810), Francisco Romero (1811-1813) y nuevamente José Gregorio Paredes (1814-1825). (Schwab, 1948: 84-86)

\section{Las fuentes}

De los tres primeros Cosmógrafos Mayores del Reino del Perú, Lucas de Quirós (1618-1634), Francisco de Quirós (1619-1645) y Diego de León (16451661), no hemos podico ubicar publicación alguna suya sobre cosmografía. Por ello, nuestro estudio iniciará a partir del cuarto de los cosmógrafos; Francisco Ruiz Lozano.

Francisco Ruiz Lozano publicó el año de 1665 una obra sobre astronomía y astrología a raíz de lo que en aquella época se creyó un cometa (en realidad se trató de una nova) y que se divisó en Lima entre fines de 1664 e inicios de 1665, dicha obra se conoce como Tratado de Cometas.

Por otro lado, a partir del arribo de Coning al cosmografiato, se inició la publicación anual de un texto en el que el Cosmógrafo Mayor del Reino daba noticias acerca del clima del año (temperaturas máximas y mínimas, lluvias, etc.), realizaba predicciones de las fases de la luna, eclipses y otros fenómenos astronómicos, publicaba tablas astronómicas calculadas al meridiano de Lima, etc. Durante el periodo de Conning se llamó Lunario. Pronostico de Temporales, y accidentes particulares de los Astros. (Sotelo, 1997:382).

A partir del arribo de Pedro de Peralta como Cosmógrafo, dicha publicación cambiará su nombre a El conocimiento de los tiempos, manteniéndose con dicho título hasta 1798. A partir de 1799 y durante el resto del periodo virreinal se llamará Almanaque peruano y guía de forasteros. (Schwab, 1948:84)

Como mencionáramos, el único de los grandes astrónomos peruanos que no llegó a ocupar el cosmografiato fue Llano y Zapata, tal vez por ser una persona sumamente culta pero autodidacta, esto es; sin educación superior formal. De éste tenemos su obra Resolución Phísico-Mathemática. Sobre la formación de los cometas, y efectos que causan sus impresiones, publicada en Lima el año de 1743. 
En lo que sigue, nos remitiremos en nuestro estudio al Tratado de Cometas de Ruiz Lozano, la Resolución Phísico-Mathemática. Sobre la formación de los cometas, y efectos que causan sus impresiones de Llano y Zapata así como a diferentes ejemplares de El Conocimiento de los tiempos y del Almanaque peruano y guía de forasteros escritos por los Cosmógrafo Mayores del Reino.

\section{Los paradigmas astronómicos}

\subsection{El paradigma ptolemaico}

\section{a) Joseph Eusebio de Llano y Zapata}

A raíz de la conquista de América la navegación de altura cobró gran importancia y como el fundamento de ésta era la astronomía, ella también adquirió una importancia bastante grande. En sus inicios, las bases teóricas de esta astronomía práctica estuvieron basadas en el sistema aristotélico-ptolemaico:

"Las bases teóricas de la astronomía práctica de la España renacentista, tenían su origen en la cosmología tradicional de origen aristotélico. La cosmografía implícita en la mayor parte de los textos publicados en aquellos años era normalmente una versión del sistema ptolemaico presentado en el Tractatu de Sphera, escrito en el siglo XIII por Joannes de Sacrobosio. Este texto presentaba de una forma clara y comprensible una simplificación del sistema geocéntrico ptolemaico y de la filosofía natural aristotélica” (Gonzáles, 1992:26-27)

Sin embargo, contrariamente a lo que a priori podríamos esperar, de los diversos astrónomos virreinales peruanos de los siglos XVII, XVIII y XIX cuyas obras hemos podido ubicar y revisar, sólo Joseph Eusebio de Llano y Zapata se nos muestra como ptolemaico, como demostraremos enseguida.

Llano y Zapata en su mencionado texto sobre los cometas, inicia su reflexión ontológica sobre ellos presentándonos la tesis clásica; aquella que suponiendo la ordenación jerárquica del cosmos en Mundo Sublunar y Mundo Supralunar, atribuía a los cometas una procedencia sublunar.

Sin embargo, a lo largo de las centurias se habían ido acumulando una gran cantidad de anomalías; eventos o sucesos astronómicos que por más que quisieran ser referidos al Mundo Sublunar, se resistían a ello. Estas anomalías (sobretodo la aparición de cometas) ${ }^{1}$ eran conocidas y aceptadas por Llano:

"Todos los cometas, que hafta eftos ultimos figlos fe han vifto, no hàn fido fublunares, fino muchos fupralunares, obfervados de los màs Doctos Mathematicos, a quienes no dàr credito, dice èl P. Efcoto, ferìa un

1 Por aquella época aún no se distinguía con exactitud entre lo que era un cometa y lo que era una súper nova. 
conocido abfurdo. Porque lo perfuaden con irrefragables razones, y argumentos deducidos de la Paralaxe de ellos." (Llano, 1743:D3)

Una alternativa a seguir a partir de la evidencia descrita era considerar a los cielos impuros aceptando que ellos pueden producir cometas, sin embargo con ello la ontología física clásica -que Llano y Zapata seguiría aceptando per se- se derrumbaría, por ello es que nuestro autor niega dicha posibilidad, presuponiendo más bien la visión clásica en su cosmología. Así, sostendrá nuestro autodidacta virreinal:

"Los cielos son incorruptibles, y lo que quiera, que se hace de su materia, tiene la misma incorruptibilidad, que ellos. El cometa siendo Cuerpo generable, y corruptible no puede formarse de materia celeste" (Llano, 1743:D1)

De este modo, por un lado Llano y Zapata seguiría aceptando la evidencia empírica sobre el lugar de los cometas en el cosmos, el cual incluía el Mundo Supralunar, pero por otro lado, aceptaría también la majestad de los cielos y su incorruptibilidad; ¿cómo conciliar ambas tesis?

Como veremos, al igual que Ruiz Lozano y a diferencia de Peralta Barnuevo, Llano y Zapata apunta hacia la alternativa más plausible para poder satisfacer ambas condiciones; son los planetas y no el cielo o éter el que produce estos fenómenos celestes que observamos en el Mundo Supralunar:

"La doctrina cierta, y segura es, que todos los Cometas, que se observan bajo de la Luna, son producidos por los halitos de la Tierra, y los que se ven más arriba, pueden ser de los efluvios de los Planetas, o vapores del Sol..." (Llano, 1743:F1)

De este modo, en consonancia con el espíritu de la tesis clásica aristotélica, los cielos son incorruptibles pero, a diferencia del Estagirita, Llano asume que los planetas son corruptibles. Esta es una modificación del esquema clásico que permite salvar los fenómenos.

En relación al sistema del mundo que seguiría Llano y Zapata, la primera pista que seguimos fue un párrafo en el que se habla de dos tipos de astros o estrellas; las fijas y las errantes o planetas:

"Las eftrellas fon de dos maneras Planetas, y Fixas. Llamanfe unas Fixas, que fon las que ocupan la fuperior parte del Cielo, y en fu movimiento dexan fiempre entresì iguales efpacios de diftancia. Planetas, ò Errantes Eftrellas fe llaman otras ... porque aunque guarden la razon cierta de los movimientos, no fe mueven con mas libertad, y en el Cielo yà aparecen en uno; yà en otro lugar" (Llano, 1743:F1)

No obstante la explicites de éste párrafo, en sí mismo es insuficiente para determinar el sistema del mundo que seguiría Llano y Zapata. 
En otro pasaje de su texto, al intentar demostrar que los cometas no son agregados de estrellas errantes o planetas (esto es, producto de conjunciones planetarias) sostiene lo siguiente:

"Los Planetas hablando comunmente tienen su curfo entro del Zodiaco, y allì exercitán fus conjunciones, oposiciones, y demàs effectos. Los Cometas fe dexan ordinariamente ver fuera de èl.

La conjuncion de los Planetas no dura mucho, como fe ven en la del Sol, y Luna: los Cometas fuelen durar algunos mefes ..." (Llano, 1743: E1)

Este pasaje parece una paráfrasis de Aristóteles, quien en los Meteorológicos sostuviera: “... todos los planetas se retardan dentro del círculo del zodíaco, mientras que muchos cometas han sido vistos fuera de ese círculo." (Aristóteles, 1996:44)

Volviendo a Llano, en este último párrafo suyo se hace inequívoca la consideración del Sol y de la Luna como unos planetas más. Y si además sólo hay estrellas fijas y planetas, y estos últimos cambian de posición con respecto a la Tierra, entonces los planetas (incluido el Sol) deben girar alrededor de la Tierra ya que ésta es tomada como punto de referencia fijo respecto a estos astros.

Si nuestra interpretación es correcta, con ello descartaríamos el que sea partidario del heliocentrismo. Llano y Zapata sería entonces un seguidor del geocentrismo.

Sobre la base de lo anterior es también posible sostener que Llano y Zapata sería un partidario de un sistema geocéntrico de filiación ptolemaica y no braheana ya que el Sol y la Luna serían para Llano y Zapata unos planetas más que girarían alrededor de la Tierra, junto con los otros planetas.

\subsection{El paradigma ticónico}

\section{a) Francisco Ruiz Lozano}

Cosmógrafo de 1662 a 1677. El catedrático sanmarquino expone sus ideas en un texto titulado "Tratado de cometas", publicado el año de 1665 y escrito con motivo de la aparición de uno de estos astros entre fines de 1664 e inicios de $1665 .^{2}$

El tradicional paradigma aristotélico-escolástico sostenía que la Tierra era el centro del universo, alrededor de ellas giraban en órbitas esféricas la Luna,

2 El texto original, en buen estilo barroco, se titula: Tratado de cometas, observacion, y ivivio del que se vio en esta civdad de los Reyes, y generalmente en todo el Mundo, por los fines del año de 1664. Y principios defte de 1665. Compuesto por el Capitan Francisco Rviz Lozano Cofmografo mayor defte Reyno, y Cathedratico de Prima de Mathematicas en efta Ciudad, tal el título completo de la obra, fue publicado por Francisco Ruiz Lozano el año de 1665 en la ciudad de Lima y consta de 42 hojas dobles de texto efectivo. 
Mercurio, Venus, Marte, Júpiter y Saturno . Esta llevó a dividir el cosmos entre el mundo sublunar, lugar de la tierra, del cambio, del devenir y la corrupción y por tanto impuro, y el mundo supralunar, lugar de los planetas y estrellas fijas, de lo inmutable, de lo perenne, de lo puro. La frontera entre estos dos mundos era la esfera lunar. Nótese que entre Venus y Marte giraba el Sol.

Estas tesis, según Ruiz Lozano, habían llevado a postular que los cometas eran formaciones originadas en el mundo sublunar por las exhalaciones de la tierra y que nunca podían remontarse más allá de ese marco, pues de lo contrario la pureza de los cielos se vería resquebrajada, por no hablar de las esferas cristalinas que sustentaban las órbitas planetarias.

Sin embargo estas tesis, sostiene Ruiz Lozano, han sido parcialmente refutadas por la observación científica de estos fenómenos durante las últimas décadas. Ruiz sostiene que el año de 1572 apareció una nueva estrella en la constelación de Casiopea. Esta estrella permaneció hasta principios de 1574. El año de 1577 hizo su aparición un cometa que, al calcular Tycho su paralaje, éste mostró que se hallaba más allá del mundo sublunar:

“... la obfervacion del Cometa del año de 1577. Que Tycho Brahe Hercules defta ciencia hizo ...

La nova de 1572. Es muy celebre, porque su duracion de dos años, y eftraña apariencia admirô a los Aftronomos, y dio materia, para que como prodigiofo la celebraffen ..." (Ruiz, 1665:10-14)

Por otro lado el perfeccionamiento del telescopio por parte de Galileo, le permitió al mismo observar más detenidamente los astros, lo cual redundó en que estableciera las manchas solares y los cráteres, o mares, lunares, aparte de las fases de Venus y los satélites de Júpiter. De esto también tenía conocimiento Ruiz Lozano:

“... determinê facar a la luz en efte tratado las ideas de los antiguos Astronomos, a quienes fijgo en parte, conformandome en lo demas con la doctrina notoriamente quilatada de los modernos, gigantes herculeos; que con nouedad la defienden, fiendo linfces celeftes (que no contentos de auer descubierto la irregularidad, oquedades, montes, mares, arrecifes, y felbas, que el curso de la Luna contiene) atalayaron las manchas; y lunares, que fe ven, y rodean el cuerpo del Sol, al modo de las ifletas, que ay en él." (Ruiz, 1665:1-2)

De este modo, Ruiz Lozano adheriría parcialmente a las tesis de Aristóteles acerca del origen sublunar de los cometas. El que hayan cometas que se puedan formar al interior de la esfera de la luna, en este caso, para él, las causas son las exhalaciones de la Tierra y no de los otros astros (Ruiz, 1665:9) por lo cual ellos pueden ser de dos clases: sublunares o supralunares (Ruiz, 1665:42) 
Establecida entonces que la pureza de los cielos no implica el que no puedan formarse y aparecer cometas en ellos ni tampoco que los plantas (incluido el Sol) puedan tener cierto tipo de manchas. Criticada también la tesis de las esferas cristalinas, se mantienen no obstante las tesis de los lugares naturales, la pureza de los cielos y el geocentrismo.

En cuanto al sistema del mundo que seguiría Ruiz Lozano, hay dos pasajes decisivos que nos han permitido establecerlo textualmente. En el primero de ellos Ruiz sostiene que los planetas describen órbitas que los alejan o acercan del centro de la Tierra:

"Sea la primera mudança de los Abfides de los Planetas, que fe mudan fegun la fuceffion de Signos, mediante la velocidad, o tardança del movimiento, haziendo tranfitos de unos lugares en otros; donde vnas vezes eftan altiffimos y apartados del centro de la Tierra, y otras muy cercanos a ellas en diferentes Signos de Zodiaco" (Ruiz, 1665:15)

Con ello queda demostrado que Francisco Ruiz Lozano era partidario del geocentrismo.

En el segundo de los pasajes se sostiene que todos los planetas (no menciona a la Tierra, por lo que se puede inferir que no la considera "planeta") están ligados al Sol y se mueven respecto a éste:

“... la mudança de la eccentricidad del Sol, a quien no fola la Venus, y Mercurio, cuyos medios movimientos fon los mifmos que el del Sol, sino los demas Planetas Saturno, lupiter, Marte, y Luna muda todos al mismo punto fus eccentricidades; porque en buena Theorica de Orbes eftan ligados al Sol de fuerte que a fu refpecto folo se mueuen.” (Ruiz, 1665:16)

Dentro del paradigma geocéntrico ambos fenómenos pueden darse únicamente en el sistema de Tycho Brahe, en éste los cinco planetas conocidos (Mercurio, Venus, Marte, Júpiter y Saturno) giran alrededor del Sol pero éste gira alrededor de la Tierra. En ese sentido las órbitas descritas por los planetas los acercan o alejan del centro de la Tierra al tiempo que se mueven alrededor del sol.

\section{b) Juan Ramón Connig}

Cosmógrafo de 1678 a 1708. En el caso de Conning no hemos podido ubicar ninguno de sus Lunarios, pero considerando sus datos biográficos, su formación académica, la filiación astronómica de su antecesor y de su sucesor en el cosmografiato y, finalmente, una famosa carta dirigida al astrónomo jesuita Atanacio Kircher (seguidor de Brahe), lo más probable es que también haya sido seguidor de Tycho Brahe.

\section{c) Pedro de Peralta Barnuevo}

Discípulo de Conning, fue Cosmógrafo desde 1709 hasta 1743. Peralta, al igual que Ruiz Lozano, estaba al tanto de los últimos descubrimientos astronó- 
micos como el de los satélites de Júpiter y Saturno, llegando a sostener "que todas hacen el numero de cinco" (Peralta, 1732: 352)

En lo referente a los satélites de Júpiter enuncia citando al mismo Galileo Galilei y a Cassini:

"Obfervò el Galilei andar al derredor del Globo de Jupiter quatro Eftrellas, que fe llamaron defpues fus Satelites, ò Archeros, por acompañarle fiempre donde va. De eftas ha calculado Monfieur Cafini los movimientos, y especialmente de la Primera..." (Peralta, 1732: 352)

También nos manifiesta que estaba al tanto de los fenómenos observados en el Sol. (Peralta, 1732: 354)

Dentro de los fenómenos celestes, el de los cometas, llamó mucho la atención de Peralta, para él, los cometas ni se crean ni se destruyen sino que serían una suerte de astros que cada cierto tiempo se acercan a nosotros y luego siguen su curso, en otras palabras, los cometas serían planetas de órbitas gigantescas. En el Canto Sexto, Octava XCVI, del Tomo II de su Lima fundada (1732) pueden leerse los siguientes versos:

"En el Cielo Phenòmeno aparece,

Que periodicos prefta lucimientos,

De donde juzgaran, fer los Cometas,

No vagas Luzes ya, reales Planetas"(Peralta, 1732: 385)

Inicialmente uno podría pensar que Peralta está influido por Newton quien también sostuviera ser los cometas planetas de órbitas gigantescas: "Si no me engaño, son una especie de planetas y retornan en órbita con un movimiento perpetuo" (Newton, 2011; 729).

Por otro lado, podría dar la impresión que Peralta, como todo poeta, utiliza indistintamente los vocablos en aras de mantener la métrica y la rima.

Sin embargo, lo anterior queda fuera de lugar al revisarse la notas explicativas que Peralta adjuntó en la publicación de los versos acabados de citar, no sólo se acepta explícitamente que los cometas sería planetas sino que además se nos explica el porqué. Peralta piensa que como los cometas aparecen periódicamente y estos periodos son más o menos exactos (como por ejemplo el del cometa Halley), ellos deben de ser planetas de órbitas gigantescas, tan grandes, que sus revoluciones no toman años sino décadas:

"De la repeticion de eftos Cometas han inferido fer verdaderos Planetas, que por la enormidad de fus Orbitas folo fe hazen vifibles fu Perigeo, ò cerca de la Tierra" (Peralta, 1732: 385)

Por otro lado, no puede adscribir al sistema heliocéntrico de Newton pues el propio Peralta, en un pasaje de la ya citada Lima fundada, escribe: "Vifte la Tierra, centro de hermosuras." (Peralta, 1732:349) 
Una interpretación literaria podría considerar que con ello simplemente alude alegóricamente al hecho de que la bóveda celeste hace creer que la Tierra se encuentra rodeada de astros que la iluminan, y nada más.

Sin embargo en un texto científico-natural como lo fue El conocimiento de los tiempos no se esperan alegorías de este tipo; ¿cómo explicar entonces afirmaciones como ésta, en el ejemplar correspondiente al año de 1743 y publicado por tanto el mismo año de su muerte? :

"Todo Paffa, y todo fe remuda: hafta el eftrago muere, y hafta el acabar tambien acaba. Hafta la permanencia de los mármoles, y la conftancia de los Montes no fon mas que bayben mas lento; y una ruina mas callada. Hasta la mifma Tierra, que es todo lo inmobil que tiene el Univerfo ..." (Peralta, 1743) ${ }^{3}$

Con esto Peralta nos está diciendo que considera que la Tierra está en el centro del Universo, como sostuvo en Lima fundada, y no lo está diciendo además de manera alegórica sino literal. Aparte de ello, la Tierra permanece inmóvil. Así pues, el sistema astronómico al que se adheriría nuestro autor sería el geocéntrico y no el heliocéntrico. Esto queda confirmado por una afirmación de Peralta del año de 1734, dos años después de publicada Lima fundada: "Pafsé à fu Tribual quando vì, que llegaba el Sol, feguido de todos los demàs Planetas..." (Peralta, 1734)

Establecida la filiación geocéntrica de Peralta, queda aún por establecer a cuál de los dos grandes sistemas geocéntricos del Universo, el del astrónomo alejandrino Claudio Tolomeo o el del noble danés Tycho Brahe, estaría siguiendo Peralta Barnuevo.

Los siguientes pasajes nos dan la clave del asunto. En el primero de éstos se sostiene que la esfera del Sol es menor que las de Júpiter y Saturno:

"Llamanfe eftos Afpectos magnos, por fer de Planetas mayores, y fuperiores al Sol, y muy tardos en fu movimiento ..." (Peralta, 1742)

Sin embargo este sólo pasaje no es suficiente ya que también en el sistema tolemaico la esfera del Sol es inferior a las de los planetas mencionados.

Un segundo pasaje, que ahora citaremos, nos orienta mejor. En éste Peralta sostiene que la Tierra no es el centro de las órbitas planetarias:

"Los Circulos ù Orbitas, que forman con su movimiento proprio los Planetas, no tienen por centro à la Tierra; y afsí fe llaman Excentricas ..." (Peralta, 1734: 351-352)

Sin embargo, en el pasaje anteriormente citado y gracias al cual se pudo establecer que Peralta no era heliocéntrico, Peralta sostenía que la Tierra era

3 Los ejemplares de El Conocimiento de los Tiempos, así como el texto que los sucedió (Almanaque peruano y guía de forasteros) no están paginados, por ello sólo citamos el autor y el año de la publicación del ejemplar. 
centro del universo ¿cómo pues compatibilizar ambos pasajes? ¿En cuál de los dos sistemas geocéntricos puede la Tierra ser el centro del universo pero no necesariamente al mismo tiempo de las órbitas planetarias, ¿en qué sistema geocéntrico es el Sol el centro de las órbitas planetarias?

De los dos sistemas geocéntricos existentes, esto sólo sucede en el sistema ticónico ya que en éste el centro de las órbitas planetarias es el propio Sol, el cual giraría alrededor de la Tierra. Esta tesis parece también ser la sostenida por Peralta Barnuevo:

"En la carrera, fi fe logrò feliz, lo que es termino, es gloria; por que comienza à correr la aprobacion, defde alli donde parò el afan. Parece que por efto duran los Magiftrados por la revoluciones del Sol, que fon los años ..." (Peralta, 1716:4)

\section{d) José de Mosquera y Villaroel (1744-1749)}

Fue Cosmógrafo desde 1744 hasta 1749. En el primer ejemplar de El conocimiento de los tiempos redactado por él (escrito en 1744 y correspondiente al año de 1745) defiende el geocentrismo y critica a Copérnico:

"La inmensa maquinaria de los Cielos, las portentosas moles de los astros ... se mueven con incesante rapidez ... Aun en la misma Tierra, a pesar de la inmovilidad de su lugar, (que negó Copernico)." (Mosquera, 1744)

Por otro lado, en ese mismo ejemplar, hablando del clima, sostiene que los planetas están, algunas veces, cerca a la Tierra y otras veces lejos de ella:

"Será en el Estío templado, y en el Invierno demasiadamente frío, porque hallándose todos los Planetas en sus Apogeos, o mayor distancia de la Tierra" (Mosquera, 1744)

Y esto es sólo posible en el geocentrismo ticónico en donde por girar los planetas alrededor del Sol y éste alrededor de la Tierra, algunas veces las órbitas planetarias alrededor del Sol, hacen que estén cercanos a la tierra y otras veces lejos de ella (dependiendo de la posición del Sol respecto a la Tierra).

\section{e) Juan Rer (1750-1756)}

Ejerció el cargo de Cosmógrafo entre 1750 y 1756 . En el ejemplar del El conocimiento de los tiempos escrito en 1751 y correspondiente al año de 1752 explicita su geocentrismo al sostener que la Tierra es la última de las esferas y ocupa además el centro del universo:

“... quife afsimifmo dejarme caer alguna véz de las fuperiores á efta infima efsphera... pues como los cuerpos graves tiran con precipitada celeridad hazia abajo, por bufcar fu centro en el de la tierra..." (Rer, 1751) 
En dicho ejemplar también menciona los problema inherentes al cálculo de la posición de los planetas desde una óptica geocéntrica y además, cita su fuente, Vicente Tosca, un conocido seguidor de Brahe:

"El trabajo de averiguar la carrera de los planetas no es tan Facil ... tiene varias anomalías ó defigualdades, una vezes andan tardos otras veloces una vezes caminan directos fegun los fignos otras retrogrados ... fuplico que vean lo que trae el Doctor Vicente Tofca ..." (Rer, 1751)

La obra de Brahe, contrariamente de lo que se piensa ahora, tuvo gran aceptación en su época pues incorporaba las últimas mediciones y observaciones pero mantenía la vieja estructura geocéntrica:

“... noble Dinamarques, y uno de los mayores Astrónomos del mundo ... siguió esta última opinión; y viendo que el Systema de Ptolomeo no podía tener lugar, á causa de no corresponder con las observaciones, compuso otro nuevo, que ha corrido con el nombre de Tychonico, y con grande aceptación en toda la Europa" (Juan, 1978: f 2)

Quien escribió estas líneas sobre Tycho Brahe es nada más que Jorge Juan, uno de los dos oficiales de la armada española que acompañó al físico y astrónomo francés Charles De la Condamine en su expedición hacia América.

Al parecer, en la época en que Jorge Juan y Antonio Ulloa publicaron sus Observaciones astronómicas, aún no estaba del todo resuelta la famosa revolución copernicana pues en su prólogo insisten que las observaciones realizadas por ellos durante su permanencia en América puede probarse la corrección del sistema heliocéntrico:

"Entre las experiencias y demostraciones Geométricas que se exponen en esta Obra, hay varias que respiran á favor del Systema conocido generalmente por el nombre de Copernicano" (Juan, 1978: f 1)

\section{f) Cosme Bueno (1757-1798)}

Asumió el cosmografiato el año de 1757 y se mantuvo en éste hasta 1798. Con él parecería que comenzaría el lento cambio en el sistema del mundo imperante en el pensamiento astronómico virreinal peruano. Pues al parecer tendría cierta influencia de Newton, lo que se puede ver tanto en algunos de sus propios textos como mediante el testimonio de sus discípulos. Así, por ejemplo, se muestra de acuerdo con las tesis del newtoniano David Gregori, en relación a los efectos negativos que podrían tener los cometas sobre la Tierra:

"Si estos vapores, añade David Gregori celebre Newtoniano, difundido por aquellos vaftos efpacios caen hacia nofotros, podrán alterar el Ayre ... caufar mucho daño al Reyno vegetal, y Animal..." (Bueno, 1756)

Por su parte, Gabriel Moreno, discípulo de Cosme Bueno y sucesor suyo en el cosmografiato sostendrá la influencia de la Teoría de la Gravitación Universal 
newtoniana en el pensamiento de su maestro, cual comenzó a tomar fuerza en el virreinato peruano a raíz de la llegada de la misión científica francesa de Charles de la Condamine, a mediados del siglo xviii:

"Hablando del físico de Newton, decía que los Académicos de Paris enviados al Perú, fueron Apóstoles del Filósofo Inglés, iniciados en lo que debían predicar en sus montañas á favor de la atracción.” (Moreno, 1799)

Por otro lado, el mismo Moreno sostiene que Cosme Bueno fue el primer astrónomo de tendencia newtoniana en el virreinato peruano: "Primer prosélito de Neuton en el Perú, adquirió la regla y exactitud de su espíritu á fuerza de estudiarlo" (Moreno, 1799)

Sin embargo, a pesar de estas referencias de su discípulo, la evidencia textual hallada por nosotros en los textos del propio Cosme Bueno no es tan contundente, pues en algunos pasajes de sus propios textos parecería discrepar de los newtonianos, así, por ejemplo, en el ejemplar El Conocimiento de los tiempos correspondiente al año de 1758 cita a un famoso astrónomo newtoniano al tiempo que expresa sus dudas sobre los cálculos de las órbitas de los cometas efectuados bajo el sistema de Newton:

"Tres especialmente fon los Cometas cuyo retorno fe predice mas ha de 50 años; que fueron el que apareció el año de 1531. el del año de 1660. y el de 1680. El primero, fegun el computo de los Neutonianos, debe volverfe a ver en el prefente de 1758 ... No falgo por fiador de fus cálculos, por motivos de larga difcufsion..” (Bueno, 1757)

En ese mismo ejemplar, en un pasaje en el cual discute cómo puede ser que un cometa ejerza un influjo negativo en la Tierra, sostiene que ello puede darse sobre todo si el cometa aparece entre el Sol y la Tierra, ya que entonces, estaría más cercano a nosotros:

"De todo lo hafta aquí brevemente dicho se colige que fi cuando fe verifica la predicción, podremos temer en los tiempos fubfequentes algunas de aquellas alteraciones que correfponden á fus malignos influxos: principalmente fi aparece entre el Sol y la tierra; pues como mas vecino entonces á nosotros ...” (Bueno, 1757)

Esto no es posible en el sistema de Ptolomeo ya que entre el Sol y la Tierra están las órbitas de la Luna, Mercurio y Venus respectivamente (los llamados "paletas interiores"). Tampoco en posible en el sistema heliocéntrico pues en éste, entre el Sol y la Tierra están las órbitas de Mercurio y Venus. Sin embargo, el que un cometa aparezca entre el Sol y la Tierra y que ello implique que dicho cometa está más cercano a nosotros que a los otros planetas sí es posible en el sistema de Tycho Brahe debido a que en éste, la Tierra ocupa el centro del cosmos y alrededor de ella primero orbita el Sol, estando los demás planetas 
orbitando alrededor del Sol. Por ello, en este sistema, si un cometa aparece entre el Sol y nosotros, obviamente está muy cercano a la Tierra.

Por lo anterior, Cosme Bueno se nos muestra más bien como partidario del geocentrismo ticónico. Así, parece que en el caso de Bueno (al igual que en sus antecesores en el cosmografiato, por lo menos hasta Ruiz), habría un marcado eclecticismo; parecería que en algunos aspectos aceptara las modernas tesis de los newtonianos pero en otros, como el sistema del mundo, seguiría siendo seguidor de Brahe. Como señalara su propio discípulo, Gabriel Moreno; "D. Cosme en su vejez ya se reía de todos los sistema" (Moreno, 1799).

Respecto a este punto podríamos argumentar que una lectura heterodoxa de los Principia de Newton, podría hallar fundamento para este eclecticismo cosmológico, por ejemplo, en el Libro III, en el capítulo titulado "Fenónenos", Newton escribe:

"Supuestas en reposo las estrellas fijas, los tiempos periódicos de los cinco planetas primarios y del Sol en torno a la Tierra o de la Tierra en torno al Sol están en razón de la potencia 3/2 de las distancias medias al Sol." (Newton, 2011:622)

En seguida Newton explica lo anterior con la siguiente frase: "Esta razón hallada por Kepler está reconocida por todos. Pues, los tiempos periódicos son los mismos y las magnitudes de las órbitas las mismas tanto si el Sol gira en torno a la Tierra como si la Tierra gira en torno al Sol." (Newton, 2011:622)

De ahí podría inferirse, para motivos de cálculo puro, aparentemente tanto la corrección de la posición kepleriana como la braheana.

En ese sentido, parecería que Cosme Bueno seguía a Newton en algunos aspectos pero en otros, principalmente el relativo a la ontología cósmica, parecería que estaba aún dentro de la tradición clásica.

\subsection{El paradigma kepleriano}

\section{a) Gabriel Moreno (1799-1809)}

Discípulo de Cosme Bueno, fue cosmógrafo entre 1799 y 1809. Es definitivamente seguidor de Newton. Así, en el ejemplar de Almanaque peruano y guía de forasteros publicado a fines de 1799 (en este año se produce el cambio de nombre de El conocimiento..., al que estamos indicando) y correspondiente al año de 1800 sostendrá que respecto a la astronomía, en los dos últimos siglos se ha llevado a cabo una revolución que ha culminado con la gravitación universal de Newton:

"En los dos últimos siglos, en que han sido tan rápidos sus progresos, debidos á la exactitud de las observaciones y aplicación de las fuerzas de Neuton á las leyes de Keplero" (Moreno, 1799) 
La relevancia del sistema kepleriano-newtoniano, por otro lado, se debe, en palabras de Moreno a "que hoy rige en la Astronomía, explicando felizmente todos los phenómenos celestes" (Moreno, 1799)

Esto lleva a que Moreno, en el ejemplar correspondiente al año de 1806, (publicado a fines del año de 1805), a reconocer los aportes de Descartes, Kepler, Leibnitz y Newton al desarrollo de las matemáticas y la astronomía:

"Descartes en la Francia, Keplero, Leibnitz en Alemania variaron el aspecto de las ciencias Exactas. La Algebra que desde su origen había dado algunos pasos adquirió baxo de la mano del primero, sino toda la perfeccion, la limpieza de sus expresiones y la aplicación a un Geometra. La Astronomia recibió del segundo las dos admirables Leyes que reynan en el movimiento de los Astros y la figura de las órbitas que corren. La Geometría debe al tercero la luz y facilidad con que el calculo diferencial penetra todas sus partes, principalmente el país inagotable de las curvas, y sus propiedades. Estos progresos guiadores de los Newton, Benouli y Euler..." (Moreno, 1805)

\section{b) Francisco Romero (1811-1813)}

Estuvo como Cosmógrafo entre los años de 1811 a 1813 por lo que tuvo a su cargo la redacción de los ejemplares del Almanaque peruano y guía de forasteros correspondientes a los años de 1812, 1813 y 1814. Sin embargo sólo hemos podido ubicar y revisar el texto escrito el año de 1813, y correspondiente por tanto al año de 1814. Lamentablemente este ejemplar, que se encuentra en la Biblioteca Nacional de Lima, está incompleto y sumamente dañado por el fuego. De lo que se puede leer, puede colegirse que sería seguidor de Newton ya que al hablar sobre los diferentes movimientos de la luna, cita a Copérnico, Newton, Halley y otros autores, mostrándose de acuerdo con éstos.

\section{c) José Gregorio Paredes (1810; 1814-1825)}

Es a la vez el último cosmógrafo del Virreinato y el primer cosmógrafo de la República del Perú ya que si bien ejerció el cargo por primera vez sólo durante un año ( fines de 1809 a fines de 1810), volverá al cosmografiato el año de 1814 y permanecerá en él hasta su muerte, el año de 1825.

En el ejemplar del Almanaque peruano y guía de forasteros correspondiente al año de 1810 y escrito el año de 1809 alaba la Ley de la Gravitación Universal:

“... la Astronomía ... viene a dividirse en geométrica y física: la una, que examina los movimientos, digámoslo así, en sí mismos: la otra ... es la que se conoce con el nombre de Mecanica celeste, y no es mas que el desarrollo del inmortal descubrimiento de la gravitación universal." (Paredes, 1809) 
Por otro lado, en el último ejemplar virreinal del Almanaque peruano y guía de forasteros, escrito a fines de 1820 y correspondiente al año de 1821 sostiene que la Tierra no es más que "un punto errante en el espacio" (Paredes, 1820)

Además sostiene que para explicar las estaciones y la diversidad de los climas no debe cometerse el error de considerar sólo las causas terrestres sino las mutaciones de la masa solar "primer principio del calor y de la constitución estacional". (Paredes, 1820)

Por lo anterior, Gregorio Paredes, último cosmógrafo del virreinato, también se nos muestra newtoniano.

\section{Causas de la persistencia de la filosofía natural clásica: algu- nas claves}

¿Por qué, mientras en Europa triunfaba la revolución copernicana hacia la segunda mitad del siglo xvii y gracias a la obra de Newton, en el virreinato peruano ésta se impondrá, al parecer, recién aproximadamente un siglo y medio después? Pensamos que por lo menos debido a las siguientes cuatro razones.

En primer lugar, pensamos que esto se debe a que los debates científicos y astronómicos suscitaban en el Perú sólo un interés utilitario ya que la astronomía había llegado de Europa y desarrollado en el Perú desde un punto de vista pragmático o aplicativo ligada especialmente a la navegación ultramarina. En ese sentido, si el viejo paradigma geocéntrico seguía funcionando en la navegación de altura ¿para qué cambiarlo?.

En segundo lugar, un lectura heterodoxa del propio texto de Newton permitía una interpretación geocéntrica de algunas de sus tesis, como ya hemos visto, por lo que los lectores americanos podían adoptar las mediciones más exactas y las herramientas de cálculo proporcionada por los Principia sin necesariamente adscribir a su compromiso ontológico heliocéntrico.

En tercer lugar, la formación impartida tanto en los colegios mayores como en la Universidad de San Marcos (que eran los lugares donde se formaba la élite intelectual del virreinato), tenía una fuerte influencia de la ciencia clásica (Martín, 2001)

En cuarto lugar, el paso del geocentrismo al heliocentrismo no era sólo un cambio de posición o reacomodo de los astros. Sacar a la Tierra del centro del universo significaba no sólo colocar al Sol en él, significaba cambiar también la concepción misma de "planeta" y a su vez la aparición de un nuevo concepto de "estrella" (diferente a las "estrellas" fijas de la última esfera). Significaba renunciar a los lugares naturales de las cosas, y a los cuatro elementos terrestres. Implicaba una nueva física, una nueva ontología y una nueva concepción del hombre y su relación con el cosmos. 
De ahí que la tesis de Tycho Brahe haya gozado, pensamos, de gran aceptación en el virreinato peruano. La solución de Brahe permitía salvar los fenómenos, adaptar un paradigma en crisis de tal modo que pueda explicar anomalías jamás previstas pero que, a pesar de ello, se mantenga vigente, si no en su forma original (el esquema de Ptolomeo es distinto del de Brahe) por lo menos sí en su espíritu (tal vez por ello Galileo asimilaba en su Diálogo sobre los dos sistemas del mundo a Brahe a la concepción tradicional de Ptolomeo). Lo otro significaba renunciar a casi dos milenios de ciencia.

\section{Conclusiones}

1. Tres fueron los paradigmas astronómicos en el virreinato peruano; el paradigma geocéntrico de Claudio Ptolomeo, el paradigma geocéntrico de Tycho Brahe y el paradigma helicéntrico kepleriano-newtoniano.

2. Hasta fines del siglo xviii convivieron los paradigmas ptolemaico y ticónico sin embargo, considerando que los Cosmógrafos Mayores (voces oficiales, en lo que astronomía de refería, en el virreinato peruano) desde Ruiz Lozano (segunda mitad del siglo xvii) hasta Cosme Bueno (fines del siglo xviii) fueron partidarios del paradigma ticónico, fue el sistema del mundo de Tycho Brahe el paradigma dominante en el virreinato peruano hasta fines del siglo xviii.

3. El representante más importante del paradigma ptolemaico en el virreinato peruano fue Joseph Eusebio de Llano y Zapata.

4. Los representantes más importantes del paradigma ticónico habrían sido Francisco Ruiz Lozano, Juan Ramón Connig, Pedro de Peralta Barnuevo, José de Mosquera y Villaroel, Juan Rer y Cosme Bueno

5. A mediados del siglo xviii en el virreinato peruano habrían comenzado a difundirse las ideas heliocéntricas, debido al aporte de la Teoría Gravitacional de Newton, así como a la misión francesa de De la Condamine.

6. El paradigma kepleriano-newtoniano se habría impuesto en el virreinato peruano a fines del siglo xviii, siendo Gabriel Moreno el primer Cosmógrafo Mayor de filiación newtoniana .

7. Los representantes más importantes del paradigma astronómico kepleriano-newtoniano en el virreinato peruano habrían sido Gabriel Moreno, Francisco Romero y Gregorio Paredes.

\section{Referencias bibliográficas}

ARISTÓTELES (1996) Los meteorológicos, Madrid: Alianza Editorial.

BUENO, Cosme (1756) El conocimiento de los tiempos, Lima.

BUENO, Cosme (1757) El conocimiento de los tiempos, Lima.

ESTREMADOYRO, Gustavo (1986) "Historia de la astronomía en el Perú" en Ernesto Ye- 
pes (Edit.) Historia de la Ciencia en el Perú. Lima: CONCYTEC y SOPHICYT, tomo I. GONZÁLES GONZÁLES, Francisco José (1992) Astronomía y navegación en España. Siglos XVI-XVIII. Madrid: Mapfre.

JUAN, Jorge y Antonio ULLOA (1978) Observaciones astronómicas y físicas hechas en los reinos del Perú, Madrid: Fundación Universitaria Española.

KUHN, Thomas (1978) La revolución copernicana. Santiago de Chile: Hyspamerica.

LLANO Y ZAPATA, Joseph Eusebio de (1743) Resolución Phísico-Mathemática. Sobre la formación de los cometas, y efectos que causan sus impresiones, Lima.

MARTÍN, Luis (2001) La conquista intelectual del virreinato del Perú. Barcelona: Casiopea.

MORENO, Gabriel (1798) El conocimiento de los tiempos, Lima.

MORENO, Gabriel (1805) Almanaque peruano y guía de forasteros. Lima.

MORENO, Gabriel (1799) Almanaque peruano y guía de forasteros, Lima.

MOSQUERA Y VILLAROEL, José de (1744) El conocimiento de los tiempos, Lima.

NEWTON, Isaac (2011) Principios matemáticos de la filosofía natural. Madrid: Alianza Editorial.

ORTIZ SOTELO, Jorge (1997) "Los Cosmógrafos Mayores del Perú en el siglo XVII" en Boletín del Instituto Riva Agüero,24; pp. 369-389.

PAREDES, Gregorio (1809) Almanaque peruano y guía de forasteros. Lima.

PAREDES, Gregorio (1820) Almanaque peruano y guía de forasteros. Lima.

PERALTA BARNUEVO Y ROCHA BENAVIDES, Pedro de (1734) El conocimiento de los tiempos, Lima.

PERALTA BARNUEVO Y ROCHA BENAVIDES, Pedro de (1742) El conocimiento de los tiempos, Lima.

PERALTA BARNUEVO Y ROCHA BENAVIDES, Pedro de (1743) El conocimiento de los tiempos, Lima.

PERALTA BARNUEVO Y ROCHA BENAVIDES, Pedro de (1732 ) Lima fundada. Lima.

PERALTA BARNUEVO Y ROCHA BENAVIDES, Pedro de (1716) Oracion que dijo el doctor D. Pedro de Peralta Barnuevo y Rocha, Contador de Cuentas y participaciones de esta Real Audiencia, y demas Tribunales de esta Ciudad por su Magestad, Cathedratico de Prima de Mathematicas, Cosmografo mayor de estos Reynos, y Rector de esta Real Universidad de San Marcos a su ilustre claustro en razon del cargo de rector, que habia ejercido, antes de la Eleccion de el, en que fue reelegido el día 30 de Junio de 1716. Lima: Universidad Nacional Mayor de San Marcos. Edición facsimilar de 1964.

RER, Juan (1751) El conocimiento de los tiempos. Lima.

RIVARA DE TUESTA, María Luisa (2000) Pensamiento Prehispánico y Filosofía colonial en el Perú. Tomo II. Lima: Fondo de Cultura Económica.

RUIZ LOZANO, Francisco (1665) Tratado de cometas, observacion, y ivivio del que se vio en esta ciudad de los Reyes, y generalmente en todo el Mundo, por los fines del año de 1664. Y principios deste de 1665. Compuesto por el Capitan Francisco Rviz Lozano Cosmografo mayor deste Reyno y Cathedratico de Prima de Mathematicas en esta Ciudad. SALAZAR BONDY, Augusto (1967) La filosofía en el Perú. Panorama histórico. Lima: Studium. SCHWAB, Federico (1948) Los almanaques peruanos: ¿1680?-1874. Lima: CIP. Separata del Boletín bibliográfico, año XXI, vol. XIX, 1-2. 